Received 07/27/2021 Review began 07/29/2021 Review ended 08/23/2021 Published 08/23/2021

(c) Copyright 2021 Bhesania et al. This is an open access article distributed under the terms of the Creative Commons Attribution License CC-BY 4.0., which permits unrestricted use, distribution, and reproduction in any medium, provided the original author and source are credited.

\section{A Case of Tick Bite Induced Babesiosis With Lyme Disease}

Siddharth Bhesania ${ }^{1}$, Kunwardeep Singh Arora ${ }^{2}$, Michal Tokarski ${ }^{1}$, Madiha Ariff ${ }^{3}$, Fastina Khan ${ }^{1}$, Janki Bhesania $^{1}$, Salman A. Haq ${ }^{1}$

1. Internal Medicine, NewYork-Presbyterian Brooklyn Methodist Hospital, Brooklyn, USA 2. Internal Medicine, Boston University School of Medicine, Boston, USA 3. Internal Medicine, Dow University of Health Sciences, Karachi, PAK

Corresponding author: Siddharth Bhesania, drsiddharthbhesania@gmail.com

\begin{abstract}
The Ixodes tick may transmit multiple pathogens, Lyme disease being the most common. Early detection of tick bites and using prophylaxis measures is the key to prevent tick bite-associated diseases like babesiosis, anaplasmosis, and Lyme disease. It is recommended to follow preventive measures like using diethyltoluamide (DEET) on the skin, applying permethrin on clothes while visiting the tick-infested areas. Co-infection is an uncommon occurrence but still representative in endemic areas. If there is delayed initiation of therapy in these kinds of patients, there may be dire consequences that may require aggressive therapy. Clinicians should consider co-infection when suspecting tick-borne disease which can prove to be fatal if not addressed promptly. Here, we present the case of a 72-year-old female with atypical symptoms, who was found to have coinfection with Lyme disease and Babesiosis on serology testing and peripheral smear and was diagnosed and treated promptly.
\end{abstract}

Categories: Internal Medicine, Infectious Disease, Epidemiology/Public Health Keywords: babesia micoti, lyme disease, ixodes tick, co-infection, pancytopenia

\section{Introduction}

Lyme disease (LD) is the most frequent infection transmitted by the Ixodes tick; however, other diseases such as babesiosis and anaplasmosis are also possible. Ticks have been attributed to illness for more than a century. The earliest recognition of illness caused by Ixodes ticks came in the early twentieth century when a Swedish dermatologist observed that the bite of an Ixodes ricinus tick was linked with erythema chronicum migrans, a distinctive skin lesion surrounding insect bites [1]. Babesiosis was first reported in humans in 1957 [2]. Since then, the number of newly identified diseases and health risks connected with Ixodes ticks has grown substantially. The incidence of dually infected Ixodes ticks appears to be higher in ticks from North American and European locations where Lyme disease is prevalent, with a reported prevalence of $\leqslant 28 \%$ [3]. Humans coinfected with LD and babesiosis appear to have more intense, prolonged symptoms than those with LD alone [3]. We present a case where a patient with atypical symptoms was found to have co-infection with Lyme disease and Babesiosis. Co-infection should be suspected if the patient presents with the atypical clinical picture of a single pathogen or lack of improvement with therapy after 48 hours for the presence of co-infection.

\section{Case Presentation}

A 72-year-old female presented with complaints of three weeks of persistent fever [maximum temperature (T-Max): 104 F], chills, nausea, and productive cough of yellow sputum. The patient traveled to Connecticut six weeks before the presentation, stayed there for 20 days. She denied any known tick bite or rash but during the previous year, she had two tick bites. She denied any other acute or chronic symptoms or any obvious sick contacts. She also denied any known immune deficiency or malignancy. She worked in a tailoring company. On initial presentation, the patient was afebrile and hemodynamically stable. Physical exam was unremarkable, with no rash, no lymphadenopathy, or evident tick bite. Initial blood work showed pancytopenia. Chest X-ray (CXR) and urinalysis (UA) were not suggestive of infection. The manual peripheral blood smear showed Babesia microti in RBCs (Figure 1). 


\section{Cureus}

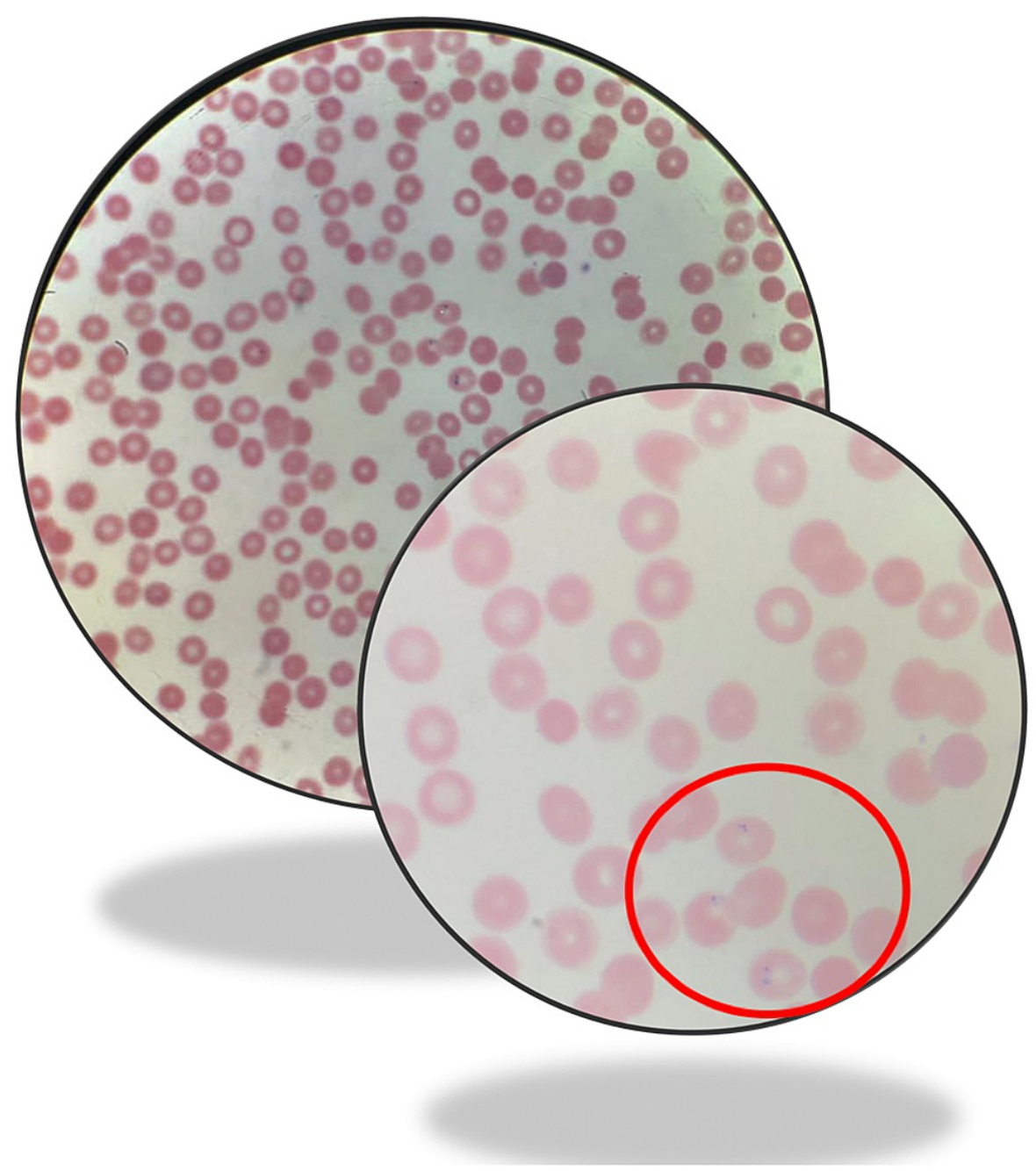

\section{FIGURE 1: Peripheral blood smear showing Babesia microti in our}

patient's blood sample.

The patient was started on azithromycin, atovaquone for babesiosis, and doxycycline to treat Lyme disease with initial suspicion of co-infection and a plan to deescalate once the Lyme disease was ruled out. Due to atypical presentation, Lyme serology and peripheral thick smear were evaluated for parasite confirmation. Lyme serology showed positive immunoglobulin M (IgM) antibodies. The patient was continued on all the antibacterial medication. Initial blood smear showed 1.7\% RBCs infected with Babesia microti which trended down to $0.4 \%$ and $<0.1 \%$ infected RBCs on repeat blood smear on days 4 and 7 . The patient was discharged home to complete the course for 14 days.

\section{Discussion}

Ixodidae have been discovered as human spotted fever vectors $[4,5]$. Spotted fever symptoms typically appear 4 to 10 days after a tick bite and vary depending on the species involved [6]. Typical symptoms include fever, headache, malaise, muscle pain, rash, and local lymphadenopathy [7]. A distinct skin lesion at the location of the tick bite, known as eschar, may develop in the majority of cases with spotted fever [7]

(Figure 2). Lyme disease can affect multiple systems of the body and produce a series of symptoms, including erythema migrans, fever, arthritis, neurologic and heart damage, and may continue to develop severe chronic consequences such as neurological symptoms and disability [8]. 


\section{Cureus}

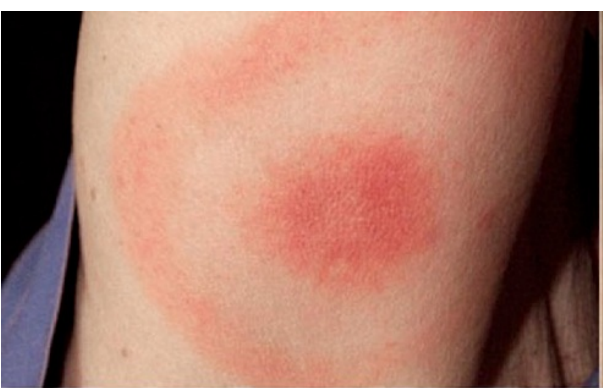

(a)

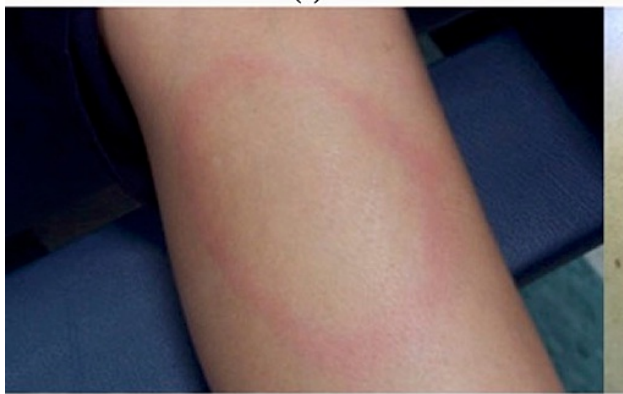

(c)

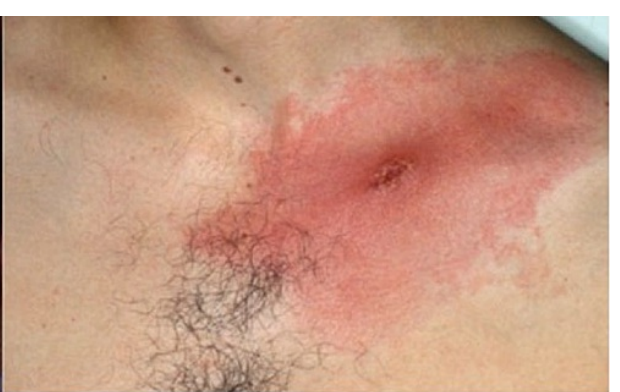

(b)

(d)

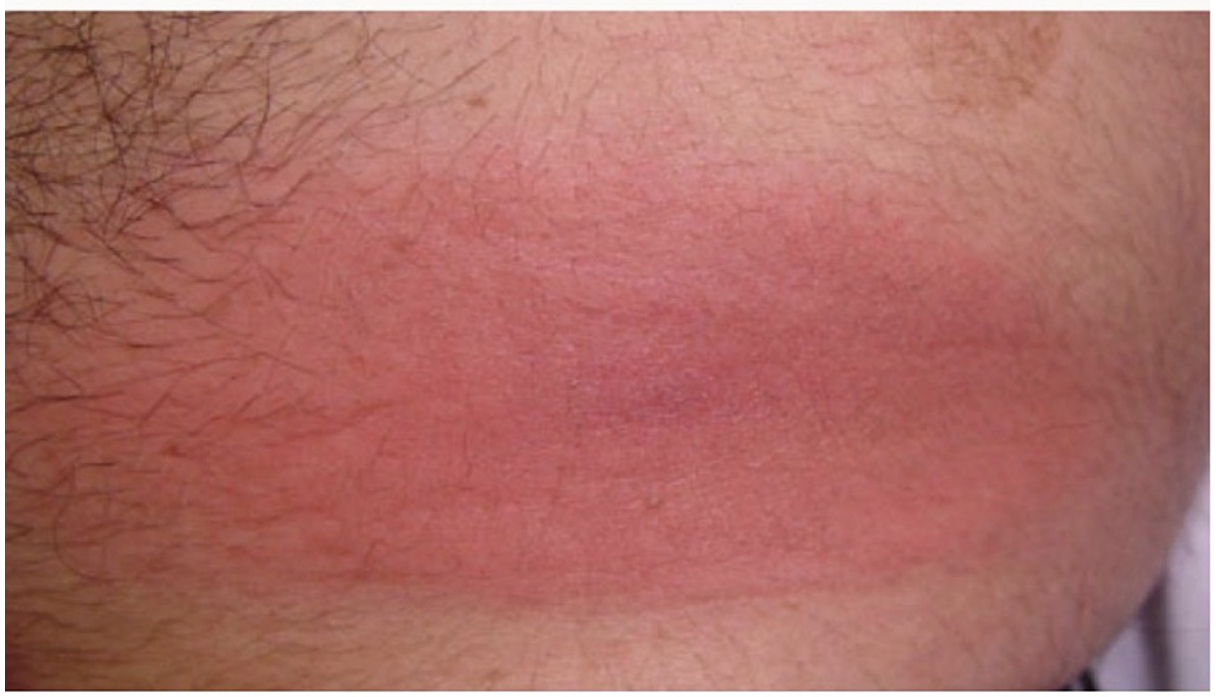

(e)

\section{FIGURE 2: Various types of rashes that can occur in Lyme disease.}

Content source for images (a) to (e): Reference [9]

(a) - Classic Lyme disease rash (circular expanding rash with target-like appearance).

(b) - Expanding rash with central crust (expanding lesion with central crust on chest).

(c) - Expanding rash with central clearing (circular, expanding rash with central clearing).

(d) - Bluish hued rash, no central clearing (Bluish hued without central clearing).

(e) - Red, oval plaque (Red, expanding oval-shaped plaque on the trunk).

The black-legged tick (Ixodes scapularis) (Figure 3) is common in the northeastern and upper midwestern United States. They are responsible for the transmission of diseases including Lyme, anaplasmosis,

babesiosis, and Powassan. The seasons with the greatest danger of tick bites are spring, summer, and fall. 


\section{Cureus}

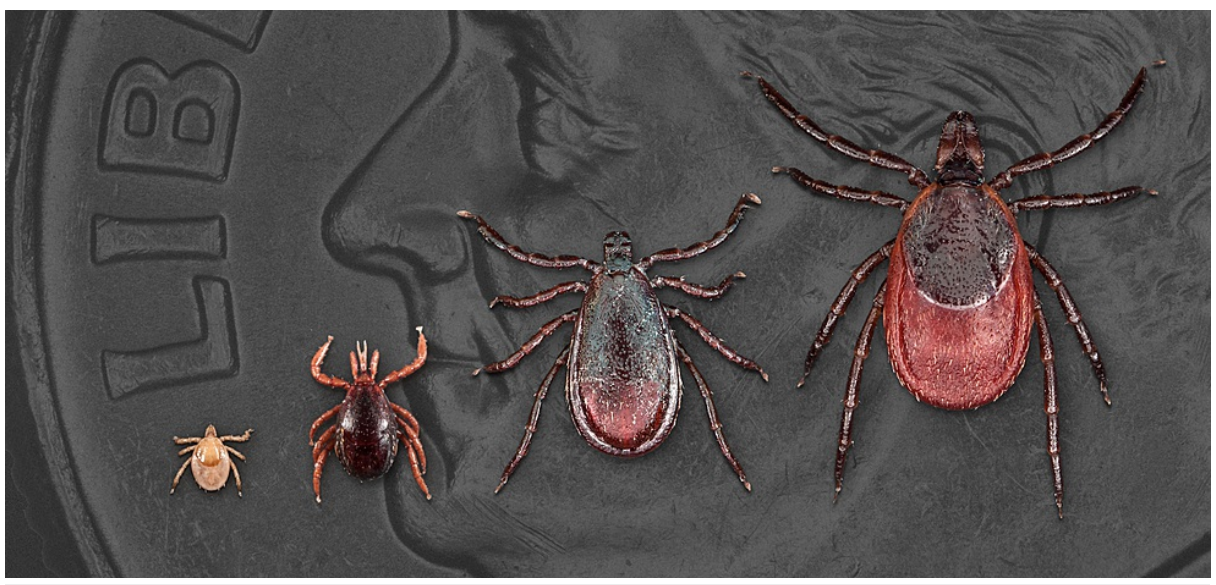

FIGURE 3: All life stages of Ixodes scapularis

Image source: Centers for Disease Control and Prevention [10]

The dime shown in the background is for scale.

When a patient comes with an adhered tick on their body, tick removal is suggested. An alcohol pretreatment is used to clean the location where the tick has adhered. It is recommended to grasp the head rather than the body, as removing the body will leave the head attached (Figure 4).

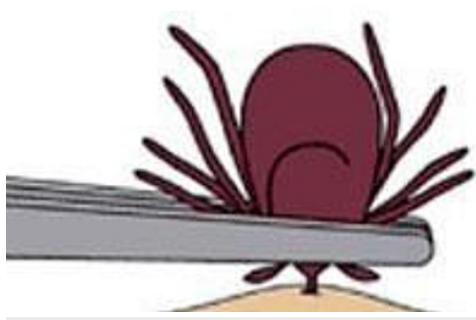

FIGURE 4: Method of tick removal from the surface of the skin

Image source: Centers for Disease Control and Prevention, National Center for Emerging and Zoonotic Infectious Diseases (NCEZID), Division of Vector-Borne Diseases (DVBD) 2019 [11].

If the head is not removed, it becomes a breeding ground for infection, and removal becomes a much more difficult task [12]. It is recommended to pull out the tick from the skin abruptly and directly while expecting the tick to take a little area of skin with it. Following removal, the skin should be cleaned routinely and a topical antibiotic, such as bacitracin, should be used, followed by application of a wound dressing to the affected region. Prophylaxis is recommended when all the following circumstances exist: (1) the attached tick is an adult or nymphal Ixodes scapularis tick, and is attached for more than 36 hours, (2) prophylaxis started within 72 hours of the time that the tick was removed, (3) the local rate of infection of these ticks with Borrelia burgdorferi is greater than $20 \%$, and (4) doxycycline is not contraindicated. Prevention of tick bites to avoid complications include: avoiding tick-infested areas, using diethyltoluamide (DEET) on the skin according to label directions, applying permethrin to clothing, wearing clothing in a downward cascade [13].

In a case report, a 67-year-old woman had a fever, rash, and myalgias for 5 days, which persisted after a 21day course of amoxicillin for Lyme disease (Borrelia burgdorferi infection). She was also found to have pancytopenia. Further workup for pancytopenia showed Babesia microti as a provocative factor for her symptoms. The patient improved after appropriate babesia treatment [14]. 
Our case has similarities with this case in that the patient had a persistent fever for three weeks along with pancytopenia. The unique feature of our case was the atypical presentation with no rash and no joint pain, but the patient had only constitutional symptoms like weakness and occasional fever, which later confirmed with positive IgM Lyme disease serology. Our patient improved with disease-specific antibacterial treatment.

\section{Conclusions}

Lyme disease is the most frequently diagnosed tick-borne disease. Patients from endemic areas may benefit from being screened for a co-infection. The clinical picture of tick-borne disease is extremely variable. Patients with delayed initiation of therapy may require aggressive therapy. Cases with severe hemolytic anemia, disseminated intravascular coagulation, respiratory failure, renal failure erythrocyte apheresis should be considered. Clinicians should consider co-infection when suspecting tick-borne disease which can lead to fatal consequences if not addressed promptly.

\section{Additional Information}

\section{Disclosures}

Human subjects: Consent was obtained or waived by all participants in this study. Conflicts of interest: In compliance with the ICMJE uniform disclosure form, all authors declare the following: Payment/services info: All authors have declared that no financial support was received from any organization for the submitted work. Financial relationships: All authors have declared that they have no financial relationships at present or within the previous three years with any organizations that might have an interest in the submitted work. Other relationships: All authors have declared that there are no other relationships or activities that could appear to have influenced the submitted work.

\section{References}

1. Afzelius A: Erythema chronicum migrans. Acta Derm Venereol. 1921, 2:120-5.

2. Skrabalo Z, Deanovic Z: Piroplasmosis in man; report of a case . Doc Med Geogr Trop. 1957, 9:11-6.

3. Swanson SJ, Neitzel D, Reed KD, Belongia EA: Coinfections acquired from ixodes ticks. Clin Microbiol Rev. 2006, 19:708-27. 10.1128/CMR.00011-06

4. La Scola B, Raoult D: Laboratory diagnosis of rickettsioses: current approaches to diagnosis of old and new rickettsial diseases. J Clin Microbiol. 1997, 35:2715-27. 10.1128/jcm.35.11.2715-2727.1997

5. Parola P, Paddock CD, Raoult D: Tick-borne rickettsioses around the world: emerging diseases challenging old concepts. Clin Microbiol Rev. 2005, 18:719-56. 10.1128/CMR.18.4.719-756.2005

6. Yoshimizu MH, Billeter SA: Suspected and confirmed vector-borne rickettsioses of North America associated with human diseases. Trop Med Infect Dis. 2018, 3:2. 10.3390/tropicalmed3010002

7. Fang R, Blanton LS, Walker DH: Rickettsiae as emerging infectious agents. Clin Lab Med. 2017, 37:383-400. 10.1016/i.cll.2017.01.009

8. Xuefei D, Qin H, Xiaodi G, et al.: Epidemiological and clinical features of three clustered cases co-infected with Lyme disease and rickettsioses. Zoonoses Public Health. 2013, 60:487-93. 10.1111/zph.12025

9. Lyme disease rashes and look-alikes. (2020). https://www.cdc.gov/lyme/signs_symptoms/rashes.html.

10. Iscap lifeseries image. https://www.cdc.gov/ticks/images/gallery/Iscap_lifeseries.jpg?noicon.

11. Tick removal. (2021). https://www.cdc.gov/ticks/removing_a_tick.html.

12. Roupakias S, Mitsakou P, Nimer AA: Tick removal. J Prev Med Hyg. 2011, 52:40-4.

13. Szczepańska A, Kiewra D, Lonc E: Influence of the fabric colour for the ticks, Ixodes ricinus and Dermacentor reticulatus attachment. Infect Dis (Lond). 2017, 49:558-60. 10.1080/23744235.2017.1293842

14. Hoversten K, Bartlett MA: Diagnosis of a tick-borne coinfection in a patient with persistent symptoms following treatment for Lyme disease. BMJ Case Rep. 2018, 2018:bcr2018225342. 10.1136/bcr-2018-225342 